\title{
Unbounded violations of bipartite Bell Inequalities via Operator Space theory
}

\author{
M. Junge ${ }^{1}$, C. Palazuelos ${ }^{2}$, D. Pérez-García ${ }^{2}$, I. Villanueva ${ }^{2}$, M.M. \\ Wolf $^{3}$ \\ 1 Department of Mathematics, University of Illinois at Urbana-Champaign, Illinois 61801- \\ 2975, USA \\ 2 Departamento de Análisis Matemático, Universidad Complutense de Madrid, 28040, Madrid, \\ Spain \\ 3 Niels Bohr Institute, 2100 Copenhagen, Denmark
}

October 22, 2009

\begin{abstract}
In this work we show that bipartite quantum states with local Hilbert space dimension $n$ can violate a Bell inequality by a factor of order $\Omega\left(\frac{\sqrt{n}}{\log ^{2} n}\right)$ when observables with $n$ possible outcomes are used. A central tool in the analysis is a close relation between this problem and operator space theory and, in particular, the very recent noncommutative $L_{p}$ embedding theory.

As a consequence of this result, we obtain better Hilbert space dimension witnesses and quantum violations of Bell inequalities with better resistance to noise.
\end{abstract}

\section{Introduction}

The fact that certain quantum correlations cannot be explained within any local classical theory is one of the most intriguing phenomena arising from quantum mechanics. It was discovered by Bell [4] as a way of testing the validity of Einstein-Podolski-Rosen's believe that local hidden variable models are a possible underlying explanation of physical reality [21]. Bell realized that the innocent looking assumptions behind any local hidden variable theory lead to non-trivial restrictions on the strength of correlations. These constraints bear his name and are since called Bell inequalities [58. Nowadays, the violation of Bell inequalities in quantum mechanics has become an indispensable tool in the modern development of Quantum Information and its applications cover a variety of areas: quantum cryptography, where it opens the possibility of getting unconditionally secure quantum key distribution [1,3, 36, 37, entanglement detection, where it is the only way of experimentally detecting entanglement without a priori hypothesis on the behavior of the experiment; complexity theory, where it enriches the theory of multipartite interactive proof systems [6, 14, 15, 24, 19, 30, 31; communication complexity (see the recent review [11]); Hilbert space dimension estimates [8, 10, 44, 56, 57]; etc. 
The violation of Bell inequalities also provides a natural way of quantifying the deviation from a local classical description. Unfortunately, computing the maximal violation for a given quantum state or Bell inequality turns out to be a daunting task except for very special cases. In 44 we uncovered a close connection between tripartite correlation Bell inequalities and the mathematical theory of operator spaces, developed since the 80's as a noncommutative version of the classical Banach space theory. With these connections at hand, and with the wide tool-box of operator spaces, we were able to prove the existence of unbounded violations of tripartite correlation Bell inequalities. At the same time this resolved an open problem in pure mathematics related to Grothendieck's famous fundamental theorem of the metric theory of tensor products. The relation of Grothendieck's theorem with correlation Bell inequalities was long ago pointed out by Tsirelson [55].

In the present paper we show how operator spaces are again the appropriate language to deal with the general bipartite case, opening in this way an avenue for the understanding of general bipartite Bell inequalities. Then, using operator space techniques, we show how to get violations of $\Omega\left(\frac{\sqrt{n}}{\log ^{2} n}\right)$, using $n$ dimensional Hilbert spaces and $k=n$ outputs. This almost closes the gap to the $\mathrm{O}(n)$ (resp. $\mathrm{O}\left(k^{2}\right)$ ) upper bound for such violations given in Proposition 2 (resp. in 18]). Again our techniques rely on probabilistic tools and use the classical random subspaces from Banach space theory which are now popular in signal processing, see [16]. The result in this paper implies the existence of better Hilbert space dimension witnesses and non-local quantum distributions with a higher resistance to noise - a desirable property when looking for loophole free Bell tests. Based on the results in [18, one can also obtains from our result new quantum-classical savings in communication complexity.

\section{Statement of the result}

We deal with the following scenario. Alice and Bob represent spatially separated observers which can choose among different observables labeled by $x=1, \ldots, N$ in the case of Alice and $y=1, \ldots, M$ in the case of Bob. The possible measurement outcomes are labeled by $a=1, \ldots, K$ for Alice and $b=1, \ldots, L$ for Bob. For simplicity we will always assume that $M=N$ and $K=L$. We will refer to the observables $x$ and $y$ as inputs and call $a$ and $b$ outputs. The object under study is the probability distribution of $a, b$ given $x, y$, that is, $P(a b \mid x y)$. Being a probability distribution, $P(a b \mid x y)$ verifies

- $P(a b \mid x y) \geq 0 \quad$ (positivity)

$-\sum_{a b} P(a b \mid x y)=1$ for all $x, y \quad$ (normalization)

In addition, we recall that a probability distribution $P=p(a b \mid x y)$ is

a) Non-signalling if

$$
\begin{aligned}
& \sum_{a} P(a, b \mid x, y)=P(b \mid y) \text { is independent of } x, \\
& \sum_{b} P(a, b \mid x, y)=P(a \mid x) \text { is independent of } y .
\end{aligned}
$$




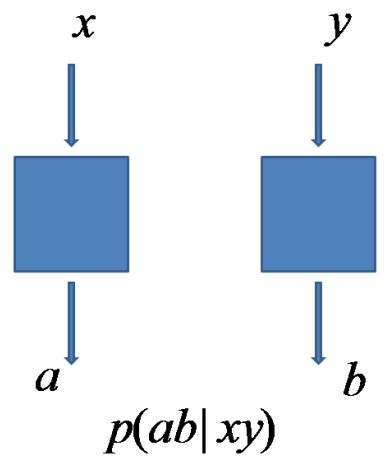

Fig. 1. $p(a b \mid x y)$ is the probability distribution of the measurement outcomes $a, b$, if Alice and Bob choose the observables labeled by $x$ and $y$ respectively.

That is, Alice choice of inputs does not affect Bob's marginal probability distribution and viceversa. This is physically motivated by the principle of Einstein locality which implies non-signalling if we assume that Alice and Bob are space-like separated. We denote the set of non-signalling probability distributions by $\mathcal{C}$.

b) Classical if

$$
P(a, b \mid x, y)=\int_{\Omega} P_{\omega}(a \mid x) Q_{\omega}(b \mid y) d \mathbb{P}(\omega)
$$

for every $x, y, a, b$, where $(\Omega, \mathbb{P})$ is a probability space, $P_{\omega}(a \mid x) \geq 0$ for all $a, x, \omega, \sum_{a} P_{\omega}(a \mid x)=1$ for all $x, \omega$ and the analogue conditions for $Q_{\omega}(b \mid y)$. We denote the set of classical probability distributions by $\mathcal{L}$.

c) Quantum if there exist two Hilbert spaces $H_{1}, H_{2}$ such that

$$
P(a, b \mid x, y)=\operatorname{tr}\left(E_{x}^{a} \otimes F_{y}^{b} \rho\right)
$$

for every $x, y, a, b$, where $\rho \in B\left(H_{1} \otimes H_{2}\right)$ is a density operator and $\left(E_{x}^{a}\right)_{x, a} \subset$ $B\left(H_{1}\right),\left(F_{y}^{b}\right)_{y, b} \subset B\left(H_{2}\right)$ are two sets of operators representing POVM measurements on Alice and Bob systems. That is, $E_{x}^{a} \geq 0$ for every $x, a, \sum_{a} E_{x}^{a}=$ $\mathbb{1}$ for every $x, F_{y}^{b} \geq 0$ for every $y, b$ and $\sum_{b} F_{y}^{b}=\mathbb{1}$ for every $y$. We denote the set of quantum probability distributions by $\mathcal{Q}$.

It is well known [55, 18] that $\mathcal{L} \varsubsetneqq \mathcal{Q} \varsubsetneqq \mathcal{C}$ and $\mathcal{C} \subset \operatorname{Aff}(\mathcal{L})$ with equality if we restrict to probability distributions. Here,

$$
\operatorname{Aff}(\mathcal{L})=\left\{\sum_{i=1}^{N} \alpha_{i} P_{i}: N \in \mathbb{N}, P_{i} \in \mathcal{L}, \alpha_{i} \in \mathbb{R}, \sum_{i=1}^{N} \alpha_{i}=1\right\}
$$

denotes the affine hull of the space $\mathcal{L}$. 
Our aim is to quantify the distance between $\mathcal{Q}$ and $\mathcal{L}$. For that, we define the 'largest Bell violation' that a given $P \in \mathcal{C}$ may attain as

$$
\nu(P)=\sup \left\{\langle M, P\rangle: M \text { verifies }\left|\left\langle M, P^{\prime}\right\rangle\right| \leq 1 \text { for every } P^{\prime} \in \mathcal{L}\right\},
$$

where $M=\left\{M_{x, y}^{a, b}\right\}_{x, y=1, a, b=1}^{N, K}$ is the "Bell inequality" acting on $P$ by duality as $\langle M, P\rangle=\sum_{x, y, a, b} P(a, b \mid x, y) M_{x, y}^{a, b}$.

Thus, in order to measure how far is the set $\mathcal{Q}$ from $\mathcal{L}$, we are interested in computing the maximal possible Bell violation

$$
\sup _{P \in \mathcal{Q}} \nu(P)
$$

Notation: In the whole paper, given a real number $x$ we write $[x]$ to denote the smallest natural number $p$ such that $x \leq p$.

Our main result states:

Theorem 1. For every $n \in \mathbb{N}$ and every $2<q<\infty$, there exists a bipartite quantum probability distribution $P$ with $\left[n^{\frac{q}{2}}\right]^{n}$ inputs per site, $n+1$ outputs and Hilbert spaces of dimension $n$ each such that

$$
\nu(P) \succeq D(q) n^{\frac{1}{2}-\frac{2}{q}},
$$

where $\succeq$ denotes inequality up to a universal constant and $D(q)$ is a constant depending only on $q$.

Actually, by the definition of $\nu$, this result is equivalent to the following dual formulation

Theorem 2. For every $n \in \mathbb{N}$ and every $2<q<\infty$, we can find a Bell inequality $M=\left(M_{x, y}^{a, b}\right)_{x, y, a, b}$, with $x, y=1, \cdots,\left[n^{\frac{q}{2}}\right]^{n}, a, b=1 \cdots, n+1$ such that

$$
\frac{\sup _{P \in \mathcal{Q}}|\langle M, P\rangle|}{\sup _{P \in \mathcal{L}}|\langle M, P\rangle|} \succeq D(q) n^{\frac{1}{2}-\frac{2}{q}} .
$$

Furthermore, the local Hilbert space dimension required to get this violation is at most $n$.

It follows from the proof of Theorem 1 given in Section 9 , that $D(q)$ can be taken to be bigger than $\frac{1}{q^{2}}$. Then, making $q=\log n$ in Theorem 1 we obtain the following

Corollary 1. For every $n \in \mathbb{N}$ there exists a bipartite quantum probability distribution $P$ with $\left[2^{\frac{\log ^{2} n}{2}}\right]^{n}$ inputs, $n+1$ outputs and Hilbert spaces each of dimension $n$ such that

$$
\nu(P) \succeq \frac{\sqrt{n}}{\log ^{2} n} .
$$

An analogous consequence holds for Theorem 2 . 


\section{Upper bounds}

We want to understand how close to optimality Theorem 1 is. In this direction, we present upper bounds to $\nu(P)$ depending on the number of outputs and the Hilbert space dimension.

First, we have the following result from [18, showing a bound for $\nu(P)$ as a function of the number of outputs.

Proposition 1. Independently of the Hilbert space dimension and the number of inputs, if $P$ is a quantum probability distribution with $k$ outputs then

$$
\nu(P)=\mathrm{O}\left(k^{2}\right) .
$$

If we fix instead the Hilbert space dimension $n$, one can prove the following proposition. A proof is provided in Appendix 2

Proposition 2. Independently of the number of inputs and outputs, if $P$ is a bipartite quantum probability distribution obtained with Hilbert spaces of local dimension $n$, then

$$
\nu(P)=\mathrm{O}(n) .
$$

\section{Prior bipartite unbounded violations}

As pointed out by Tsirelson [55], Grothendieck's Theorem, which he himself called the fundamental theorem of the metric theory of tensor products, shows that we can not obtain unbounded violations in the case of correlation matrices.

The first unbounded violations of Bell inequalities can be traced back to an application of Raz parallel repetition theorem 50, which trivially ensures that the parallel repetition of the magic square game has a violation which grows with $n$ inputs, $n$ outputs and a Hilbert space of dimension $n$ as $n^{x}$ for some $x>0$. Similar results hold for any pseudo-telepathy game 7 . Even using the improved version of Raz theorem given recently in [23 49, or the concentration theorem given in [49, the best nowadays available lower bound using this technique seems to be not much better than $\Omega\left(n^{10^{-5}}\right)$.

In [30, the authors make a spectacular improvement over this last quantity. They prove the existence, for each $\nu$, of unique two provers one round games with $n$ outputs and $2^{n} / n$ inputs such that the quantum value of the game is larger than $1-54 \nu$ and the classical one smaller than $2 / n^{\nu}$. This involves a violation of order $\Omega\left(n^{\frac{1}{54}}\right)$. Their proof strongly relies on a deep result of Khot and Vishnoi in the context of complexity theory [32].

Therefore, our $\Omega\left(\frac{\sqrt{n}}{\log ^{2} n}\right)$ violation with $n$ outputs and local Hilbert space dimension $n$ can be seen as an important improvement to the previous results. The prize to pay is the increase of the number of inputs to $\mathrm{O}\left(\left[2^{\frac{\log ^{2} n}{2}}\right]^{n}\right)$.

\section{Resistance to noise}

In the search for a loophole free Bell test, much has been written about nonlocality in the presence of detector inefficiencies (see for instance [9, 11, 12, 13, 38, 
39, 43]). This is modelled in 38 by adding an extra output $\perp$ that means "no detection" in both Alice and Bob sides. If the detector efficiency is $\eta$, we then change the "perfect" probability distribution $P=P(a b \mid x y)$ by $\eta^{2} P+\left(1-\eta^{2}\right) P^{\prime}$ where $P^{\prime}=P^{\prime}(a b \mid x y) \in \mathcal{L}$ is the local distribution defined by

$\left(1-\eta^{2}\right) P^{\prime}(a b \mid x y)=\eta(1-\eta) P(a \mid x) \delta_{b, \perp}+\eta(1-\eta) \delta_{a, \perp} P(b \mid y)+(1-\eta)^{2} \delta_{a, \perp} \delta_{b, \perp}$.

That is, we can interpret the inefficiency of the detector as a local noise added to the original probability distribution. The same happens with other classes of imperfections in the detectors: for instance if, with certain probability, the detector produces a random output instead of working properly.

Therefore, in order to have non-local distributions even in the presence of noise, we fix $P \in \mathcal{C}$ and look at

$$
\pi(P)=\inf \left\{\pi: \text { for all } P^{\prime} \in \mathcal{L}, \pi P+(1-\pi) P^{\prime} \notin \mathcal{L}\right\} .
$$

The following proposition shows that this is "exactly" what we are estimating. Specifically,

Proposition 3. For every $P \in \mathcal{C}$,

$$
\nu(P)=\frac{2}{\pi(P)}-1 .
$$

By our main result, this proves the existence of quantum probability distributions with $n$ outputs and Hilbert spaces of dimension $n$ which can withstand any local noise with relative strength $O\left(1-\frac{\log ^{2}(n)}{\sqrt{n}}\right)$ (see next section). It is interesting to note that, by Proposition 2 , $\left(1-\frac{1}{n}\right)$ is an upper bound for the maximal possible resistance to noise. However, if one restricts exclusively to the noise coming from inefficient detectors, one can obtain exponential resistance [38]. It is time for the proof of Proposition 3.

Proof. Let $P \in \mathcal{C}$. We refer to [18] for the fact that

$$
\nu(P)=\inf \left\{\sum_{i=1}^{I}\left|\alpha_{i}\right|: P=\sum_{i=1}^{I} \alpha_{i} P_{i}, P_{i} \in \mathcal{L}, \alpha_{i} \in \mathbb{R}, \sum_{i=1}^{I} \alpha_{i}=1\right\} .
$$

Let $\lambda=\pi(P)$. By definition we have $\lambda P+(1-\lambda) P^{\prime}=P^{\prime \prime}$ is again in $\mathcal{L}$. This gives $P=\frac{1}{\lambda} P^{\prime \prime}-\left(\frac{1}{\lambda}-1\right) P^{\prime}$ and therefore, by Equation (4), $\nu(P) \leq \frac{2}{\lambda}-1$.

For the converse we use again Equation (4) and start with the decomposition $P=\sum_{i} \alpha_{i} P_{i}$ such that $\sum_{i}\left|\alpha_{i}\right|=\nu(P)$. Dividing in positive and negative terms we get $P=\sum_{i} \alpha_{i}^{+} P_{i}-\sum_{i} \alpha_{i}^{-} P_{i}$, where $\sum_{i} \alpha_{i}^{+}-\sum_{i} \alpha_{i}^{-}=1$ and $\sum_{i} \alpha_{i}^{+}+$ $\sum_{i} \alpha_{i}^{-}=\nu(P)$. Let us denote by $r=\sum_{i} \alpha_{i}^{+}$the positive part. Hence we have $2 r=\nu(P)+1$ and therefore

$$
\frac{1}{r} P+\left(1-\frac{1}{r}\right) P^{\prime}=P^{\prime \prime}, P^{\prime}, P^{\prime \prime} \in \mathcal{L}
$$

Indeed, $P^{\prime}=\frac{\sum_{i} \alpha_{i}^{-} P_{i}}{\sum_{j} \alpha_{j}^{-}}$and $P^{\prime \prime}=\frac{\sum_{i} \alpha_{i}^{+} P_{i}}{\sum_{j} \alpha_{j}^{+}}$. Equation 5 gives $\lambda \geq \frac{1}{r}$. Since $2 r=\nu(P)+1$ we obtain that $\nu(P) \geq \frac{2}{\lambda}-1$, which concludes the proof. 


\section{Incomplete probability distributions}

We present here incomplete probability distributions. We need them for the statement and proof of Theorem 3. Our main result, Theorem[1, will follow as a corollary. We also use incomplete probability distributions to formalize the treatment given to noise in the previous section.

We are interested in computing $\pi(P)$ when we consider local probability distributions $P^{\prime}$ with $k+1$ outputs in Equation (3), where $k$ is the number of outputs of $P$. To this end we embed $P$ into the space of probability distribution of $k+1$ outputs just by adding the corresponding 0's and denoting the new distribution by $\tilde{P}$. We denote $\mathcal{L}_{k}$ to the local distributions with $k$ outputs (the other parameters are fixed). By Proposition 3, we compute

$$
\nu(\tilde{P})=\sup _{M} \frac{|\langle M, \tilde{P}\rangle|}{\sup _{P^{\prime} \in \mathcal{L}_{k+1}}\left|\left\langle M, P^{\prime}\right\rangle\right|} .
$$

Of course, restricting with $M$ 's which vanish on the index given by the extra output $\perp$ will give a lower bound for $\nu(\tilde{P})$. That is, we have

$$
\nu(\tilde{P}) \geq \sup _{M} \frac{|\langle M, P\rangle|}{\sup _{P^{\prime} \in \mathcal{L}_{k}}\left|\left\langle M, P^{\prime}\right\rangle\right|},
$$

where $P^{\prime}$ is now of the form

$$
P^{\prime}(a, b \mid x, y)=\int_{\Omega} P_{\omega}(a \mid x) Q_{\omega}(b \mid y) d \mathbb{P}(\omega) .
$$

$(\Omega, \mathbb{P})$ is a probability space and for every $\lambda, x$ (resp. $y)(P(a \mid x, \omega))_{x}^{a}$ (resp. $\left.(Q(b \mid y, \omega))_{y}^{b}\right)$ is a sequence of positive numbers such that $\left.\sum_{a} P(a \mid x, \omega)\right)_{x}^{a} \leq 1$ (resp. is $\left.\left.\sum_{a} Q(a \mid x, \omega)\right)_{x}^{a} \leq 1\right)$. We will say that such a $P^{\prime}$ is an incomplete classical probability distribution.

In this section we deal with this kind of incomplete probability distributions and prove a generalization of our main result, Theorem 1 to this setting. This will formalize the claim stated in Section 5 concerning the existence of quantum probability distributions with $n$ outputs and Hilbert spaces of dimension $n$ which can withstand any local noise with extra outputs and relative strength $O(1-$ $\left.\frac{\log ^{2}(n)}{\sqrt{n}}\right)$.

The rest of the paper is essentially devoted to prove the above mentioned generalization, from which Theorem 1 can be deduced.

We say that $P$ is an incomplete quantum probability distribution if there exist two Hilbert spaces $H_{1}, H_{2}$ such that

$$
P(a, b \mid x, y)=\operatorname{tr}\left(E_{x}^{a} \otimes F_{y}^{b} \rho\right)
$$

for every $x, y, a, b$, where $\rho \in B\left(H_{1} \otimes H_{2}\right)$ is a density operator and $\left(E_{x}^{a}\right)_{x, a} \subset$ $B\left(H_{1}\right),\left(F_{y}^{b}\right)_{y, b} \subset B\left(H_{2}\right)$ are two sets of operators representing incomplete POVM measurements on Alice and Bob systems. That is, $E_{x}^{a} \geq 0$ for every $x, a, \sum_{a} E_{x}^{a} \leq \mathbb{1}$ for every $x, F_{y}^{b} \geq 0$ for every $y, b$ and $\sum_{b} E_{y}^{b} \leq \mathbb{1}$ for every $y$.

We denote the set of incomplete quantum distributions by $\mathcal{Q}^{\text {in }}$ and the set of incomplete classical distributions by $\mathcal{L}^{i n}$.

With these definitions at hand, we can introduce 
Definition 1. Given a linear functional (Bell inequality) $M=\left(M_{x, y}^{a, b}\right)_{x, y=1, a, b=1}^{N, K}$, we define the Classical bound of $M$ as the number

$$
B_{C}(M)=\sup \left\{|\langle M, P\rangle|: P \in \mathcal{L}^{i n}\right\}
$$

and the Quantum bound of $M$ as

$$
B_{Q}(M)=\sup \left\{|\langle M, P\rangle|: P \in \mathcal{Q}^{i n}\right\} .
$$

We define the largest quantum violation of $M$ as the positive number

$$
L V(M)=\frac{B_{Q}(M)}{B_{C}(M)}
$$

Remark 1. It is easy to see that $B_{C}(M)=0$ implies $B_{Q}(M)=0$ for every $M$. We will rule out these cases because they lack interest.

The generalization of Theorem 1 to this context is the following one.

Theorem 3. For every $n \in \mathbb{N}$ and every $2<q<\infty$, we can find a linear functional $M=\left(M_{x, y}^{a, b}\right)_{x, y, a, b}, x, y=1, \cdots,\left[n^{\frac{q}{2}}\right]^{n}, a, b=1 \cdots, n$ such that

$$
L V(M) \succeq D(q) n^{\frac{1}{2}-\frac{2}{q}} .
$$

The local Hilbert space dimension required to get this violation is at most $n$.

Our next two results follow straightforwardly.

Corollary 2. For every $n \in \mathbb{N}$ we can find a linear functional $M=\left(M_{x, y}^{a, b}\right)_{x, y, a, b}$, $x, y=1, \cdots,\left[2^{\frac{\log ^{2} n}{2}}\right]^{n}, a, b=1 \cdots, n$ such that

$$
L V(M) \succeq \frac{\sqrt{n}}{\log ^{2} n} .
$$

The local Hilbert space dimension needed to get this violation is at most $n$.

Corollary 3. For all $n$, there exists a probability distribution $P$ with $\left[2^{\frac{\log ^{2} n}{2}}\right]^{n}$ inputs, $n+1$ outputs and Hilbert space dimension $n$ which can withstand any local noise with extra outputs and relative strength $O\left(1-\frac{\log ^{2} n}{\sqrt{n}}\right)$.

Finally, the next lemma allows us to prove Theorem 2 (and, thus, Theorem 1) from Theorem 3

Lemma 1. Suppose we have a linear functional $\left(M_{x, y}^{a, b}\right)_{x, y, a, b}, x, y=1, \cdots, N$, $a, b=1, \cdots, K$ such that $L M(V)=C$. Then, there exists another linear functional $\left(\hat{M}_{x, y}^{a, b}\right)_{a b x y}, x, y=1, \cdots, N, a, b=1, \cdots, K+1$ such that

$$
\frac{\sup _{P \in \mathcal{Q}}|\langle\hat{M}, P\rangle|}{\sup _{P \in E}|\langle\hat{M}, P\rangle|}=C \text {. }
$$

Proof. It is enough to define $\hat{M}$ as the extension of $M$ for which $\hat{M}_{x, y}^{K+1, b}=0$, $\hat{M}_{x, y}^{a, K+1}=0$. 


\section{Bounds for the Hilbert space dimension}

The interest in testing the Hilbert space dimension started with a crucial observation made in 3 . In that paper, the authors observe that the standard security proofs for the BB84 protocol [53,33. assume a given dimension in the Hilbert space and they can fail if this assumption is dropped. In 10, motivated by that, the authors define the concept of "dimension witness" and show some examples in low dimensions. Since then, several contributions to the field have appeared with different approaches: Bell inequalities [8, 56, quantum random access codes 57. or quantum evolutions 59 .

We define $\mathcal{Q}_{d}$ to be the distributions in $\mathcal{Q}$ with the extra restriction that the Hilbert spaces $H_{1}, H_{2}$ appearing in the definition are $d$-dimensional. With this notation, a dimension witness for dimension $d$ is simply a "Bell inequality" $M_{d, n}$ such that $\left|\left\langle M_{d, n}, P_{d}\right\rangle\right| \leq C_{d}$ for all $P_{d} \in \mathcal{Q}_{d}$, and for such that there exists $P \in \mathcal{Q}_{n}$ with $\left|\left\langle M_{d, n}, P\right\rangle\right|>C_{d}$. In the case of binary outcomes, Briët, Buhrman and Toner 8 and Vertesi and Pal 56 have shown how to get dimension estimates for any dimension. However, in their case

$$
\sup _{M_{d, n}} \frac{\sup _{P_{n} \in \mathcal{Q}_{n}}\left|\left\langle M_{d, n}, P_{n}\right\rangle\right|}{\sup _{P_{d} \in \mathcal{Q}_{d}}\left|\left\langle M_{d, n}, P_{d}\right\rangle\right|} \in\left[1, K_{G}\right] .
$$

This means that the resolution of the considered witnesses is bounded by Grothendieck's constant $K_{G}$ and indeed could vanish with increasing dimension.

It would be therefore desirable to get

$$
\sup _{M_{d, n}} \frac{\sup _{P_{n} \in \mathcal{Q}_{n}}\left|\left\langle M_{d, n}, P_{n}\right\rangle\right|}{\sup _{P_{d} \in \mathcal{Q}_{d}}\left|\left\langle M_{d, n}, P_{d}\right\rangle\right|} \underset{n \geq d \rightarrow \infty}{\longrightarrow} \infty .
$$

For two outcomes this was shown to be possible in the tripartite case [44]. Our main Theorem, together with Theorem 2 implies that

Theorem 4. For any $d, n$ we can define dimension estimates $M_{d, n}$ verifying

$$
\sup _{M_{d, n}} \frac{\sup _{P_{n} \in \mathcal{Q}_{n}}\left|\left\langle M_{d, n}, P_{n}\right\rangle\right|}{\sup _{P_{d} \in \mathcal{Q}_{d}}\left|\left\langle M_{d, n}, P_{d}\right\rangle\right|}=\Omega\left(\frac{\sqrt{n}}{\log ^{2}(n) d}\right) .
$$

\section{Mathematical tools and Connections}

In this section we will introduce the basic notions about operator spaces which we will need along this work. We do recommend [20] and [46] for a much more complete reference.

The theory of operator spaces was born with the work of Effros and Ruan in the 80 's, see for instance [20,46. They characterized, in an abstract sense, the structure of the closed subspaces of $B(H)$, the space of bounded linear operators on a Hilbert space.

Formally, an operator space is a complex vector space $E$ and a sequence of norms $\|\cdot\|_{n}$ in the space of $E$-valued matrices $M_{n}(E)=M_{n} \otimes E$, which verify the following two properties 
1. For every $n, m \in \mathbb{N}, x \in M_{m}(E), a \in M_{n m}$ and $b \in M_{m n}$ we have that

$$
\|a x b\|_{n} \leq\|a\|\|\| x\left\|_{m}\right\| b \|
$$

2. For every $n, m \in \mathbb{N}, x \in M_{n}(E), y \in M_{m}(E)$, we have that

$$
\left\|\left(\begin{array}{ll}
x & 0 \\
0 & y
\end{array}\right)\right\|_{n+m}=\max \left\{\|x\|_{n},\|y\|_{m}\right\} .
$$

Any $C^{*}$-algebra $\mathcal{A}$ has a natural operator space structure induced by its natural embedding $j: \mathcal{A} \hookrightarrow B(H)$. Indeed, it is enough to consider the sequence of norms on $M_{n} \otimes \mathcal{A}$ defined by the embedding $i d \otimes j: M_{n} \otimes \mathcal{A} \hookrightarrow M_{n} \otimes$ $B(H)=B\left(\ell_{2}^{n} \otimes_{2} H\right)$. In particular, $\ell_{\infty}^{k}$ has a natural operator space structure. To compute it we isometrically embed $\ell_{\infty}^{k}$ into the diagonal of $M_{k}$ and then, given $x=\sum_{i} A_{i} \otimes e_{i} \in M_{n}\left(\ell_{\infty}^{k}\right)=M_{n} \otimes \ell_{\infty}^{k}$, we have

$$
\|x\|_{n}=\| \sum_{i} A_{i} \otimes|i\rangle\langle i|\left\|_{M_{n k}}=\max _{i}\right\| A_{i} \|_{M_{n}} .
$$

In order to attain a better understanding of the differences between Banach space category and operator space category, we need to look not only at the spaces, but also at the morphisms, that is, the operations which preserves the structure. We will have to consider now the so called completely bounded maps. They are linear maps $u: E \longrightarrow F$ between operator spaces such that all the dilations $u_{n}=\mathbb{1}_{n} \otimes u: M_{n} \otimes E=M_{n}(E) \longrightarrow M_{n} \otimes F=M_{n}(F)$ are bounded. The cb-norm of $u$ is then defined as $\|u\|_{c b}=\sup _{n}\left\|u_{n}\right\|$. We will call $C B(E, F)$ the resulting normed space. It has a natural operator space structure induced by $M_{n}(C B(E, F))=C B\left(E, M_{n}(F)\right)$. We can analogously define the notion of a complete isomorphism/isometry (see [20,46]).

The so called minimal tensor product of two operator spaces $E \subset B(H)$ and $F \subset B(K)$ is defined as the operator space $E \otimes_{\min } F$ with the structure inherited from the induced embedding $F \otimes E \subset B(H \otimes K)$. In particular, $M_{n}(E)=$ $M_{n} \otimes_{\min } E$ for every operator space $E$. The tensor norm min in the category of operator spaces will play the role of the so called $\epsilon$ norm in the classical theory of tensor norms in Banach spaces 17. In particular min is injective, in the sense that if $E \subset X$ and $F \subset Y$ completely isomorphic/isometric, then $E \otimes_{\min } F \subset X \otimes_{\min } Y$ completely isomorphic/isometric. The analogue in the operator space category of the $\pi$ tensor norm is the projective tensor norm, defined as

$$
\|u\|_{M_{n}\left(E \otimes^{\wedge} F\right)}=\inf \left\{\|\alpha\|_{M_{n, l m}}\|x\|_{M_{l}(E)}\|y\|_{M_{m}(F)}\|\beta\|_{M_{l m, n}}: u=\alpha(x \otimes y) \beta\right\},
$$

where $u=\alpha(x \otimes y) \beta$ means the matrix product

$$
u=\sum_{r s i j p q} \alpha_{r, i p} \beta_{j q, s}|r\rangle\langle s| \otimes x_{i j} \otimes y_{p q} \in M_{n} \otimes E \otimes F .
$$

Both tensor norms, $\wedge$ and min, are associative and commutative and they share the duality relations which verify $\pi$ and $\epsilon$ in the context of Banach spaces. In 
particular, for finite dimensional operator spaces we have the natural completely isometric identifications

$$
\left(E \otimes^{\wedge} F\right)^{*}=C B^{2}(E, F ; \mathbb{C})=C B\left(E, F^{*}\right)=E^{*} \otimes_{\min } F^{*},
$$

where, given an operator space $E$, we define its dual operator space $E^{*}$ via the identification $M_{n}\left(E^{*}\right)=C B\left(E, M_{n}\right)$.

Given a Banach space $X$, we can consider in it different operator space structures or, equivalently, different isometric embeddings of $X$ into $B(H)$ which lead to different families of matrix norms. For example we may embed an $n$ dimensional Hilbert space as column

$$
C_{n}=\left\{\sum_{k} \alpha_{k}|k\rangle\langle 0|: \alpha_{k} \in \mathbb{C}\right\} \quad \text { or row space } \quad R_{n}=\left\{\sum_{k} \alpha_{k}|0\rangle\langle k|: \alpha_{k} \in \mathbb{C}\right\}
$$

Let us note that

$\left\|\sum_{i} A_{i} \otimes e_{i}\right\|_{M_{m} \otimes_{\min } R_{n}}=\left\|\sum_{i} A_{i} A_{i}^{\dagger}\right\|^{\frac{1}{2}},\left\|\sum_{i} A_{i} \otimes e_{i}\right\|_{M_{m} \otimes_{\min } C_{n}}=\left\|\sum_{i} A_{i}^{\dagger} A_{i}\right\|^{\frac{1}{2}}$.

Using matrices of the form $A_{i}=|i\rangle\langle 0|$ we deduce the well-known fact that this yields different matrix norms (see [46] from more details).

The natural operator space structure on $\ell_{1}^{n}$ is the one obtained by the $\mathrm{du}-$ ality $\left(\ell_{\infty}^{n}\right)^{*}=\ell_{1}^{n}$ and it can be seen that for every operator space $X$ the space $\ell_{\infty} \otimes_{\min } X$ (resp. $\left.\ell_{1} \otimes^{\wedge} X\right)$ coincides, as a Banach space, with $\ell_{\infty} \otimes_{\epsilon} X=\ell_{\infty}(X)$ (resp. $\ell_{1} \otimes_{\pi} X=\ell_{1}(X)$ ). Furthermore, for every operator space $X$, the natural operator space structure defined on $\oplus_{1}^{n} X$ (see [46]) allows us to identify completely isometrically this operator space with $\ell_{1}^{n} \otimes^{\wedge} X$ via the natural identification. This operator space is denoted by $\ell_{1}^{n}(X)$. Analogous reasonings hold for the operator space $\ell_{\infty}^{n}(X)$. Actually, by the comments above, it follows that

$$
\left(\ell_{\infty}^{n}(X)\right)^{*}=\ell_{1}^{n}\left(X^{*}\right) \text { (completely isometrically) }
$$

for every finite dimensional operator space $X$.

The operator space $L_{p}$-embedding theory has been developed in the last years. Some of the most important results of classical Banach space theory, as well as probability theory and harmonic analysis have found analogous versions in the noncommutative case [29,26, 27].

As we did in a previous work [4], we will reduce the problem of separating the Classical from the Quantum probability distributions to the problem of separating the epsilon and the min norm on the tensor products of certain operator spaces (see Section 8.1). The noncommutative $L_{p}$-embedding theory will allow us to find "good" subspaces where we can compute the above mentioned tensor norms.

Our new tool in this paper are spaces constructed as sums and intersection in interpolation theory. These spaces already play an important role for embedding 
problems in operator space theory 28,27 . For fixed $t>0$ and $m \in \mathbb{N}$, we consider the operator space $K\left(t ; \ell_{\infty}^{m}, R^{m}+C^{m}, \ell_{1}^{m}\right)$ defined by the matrix norm

$$
\begin{aligned}
& \|x\|_{M_{n}\left(K\left(t ; \ell_{\infty}^{m}, R^{m}+C^{m}, \ell_{1}^{m}\right)\right)} \\
& =\inf _{x=x_{1}+x_{2}+x_{3}}\left\{\left\|x_{1}\right\|_{M_{n}\left(\ell_{\infty}^{m}\right)}+\sqrt{t}\left\|x_{2}\right\|_{M_{n}\left(R^{m}+C^{m}\right)}+t\left\|x_{3}\right\|_{M_{n}\left(\ell_{1}^{m}\right)}\right\}
\end{aligned}
$$

As in classical interpolation theory [28, Lemmas 3.1, 3.5], it is easy to determine the dual space:

$$
K\left(t ; \ell_{\infty}^{m}, R^{m}+C^{m}, \ell_{1}^{m}\right)^{*} \sim J\left(t^{-1} ; \ell_{1}^{m}, R^{m} \cap C^{m}, \ell_{\infty}^{m}\right),
$$

where $J\left(t^{-1} ; \ell_{1}^{m}, R^{m} \cap C^{m}, \ell_{\infty}^{m}\right)$ denotes the operator space given by

$$
\begin{aligned}
& \|a\|_{M_{n}\left(J\left(t^{-1} ; \ell_{1}^{m}, R^{m} \cap C^{m}, \ell_{\infty}^{m}\right)\right)} \\
& =\max \left\{\|a\|_{M_{n}\left(\ell_{1}^{m}\right)}, t^{-\frac{1}{2}}\|a\|_{M_{n}\left(R^{m} \cap C^{m}\right)}, t^{-1}\|a\|_{M_{n}\left(\ell_{\infty}^{m}\right)}\right\} .
\end{aligned}
$$

Here, $\sim$ denotes a complete isomorphism up to a universal constant (in this case 16). The following result will be crucial in our work:

Theorem 5 ([28], Theorem 3.6). Let $(\Omega, \mu)$ be a measure space such that $\mu(\Omega)=n$. Then, the application

$$
j: L_{1}(\Omega)+L_{2}^{r}(\Omega)+L_{2}^{c}(\Omega)+L_{\infty}(\Omega) \hookrightarrow L_{1}\left(\Omega^{n} ; \ell_{\infty}^{n}\right),
$$

defined by

$$
j(f)\left(\omega_{1}, \cdots, \omega_{n}\right)=\frac{1}{n^{n}} \sum_{k=1}^{n} f\left(\omega_{k}\right) e_{k}
$$

is a complete embedding (with absolute constants).

This result is stated in a much more general context in [28. In Appendix A.2 we boil the rather heavy notation down to the result used here.

8.1. Connection to the "min vs $\varepsilon$ problem". In this section we will connect the Classical (resp. Quantum) bounds of a given linear functional $M$ (see Definition 1) to two natural tensor norms in the framework of classical Banach spaces and operator spaces.

We associate with a four dimensional matrix with coefficients $M=\left(M_{x, y}^{a, b}\right)_{x, y=1, a, b=1}^{N, K}$ the corresponding tensor

$$
\sum_{x, y=1, a, b=1}^{N, K} M_{x, y}^{a, b}\left(e_{x} \otimes e_{a}\right) \otimes\left(e_{y} \otimes e_{b}\right)
$$

considered as an element of $\ell_{1}^{N}\left(\ell_{\infty}^{K}\right) \otimes \ell_{1}^{N}\left(\ell_{\infty}^{K}\right)$. Our next result deals with the Classical bound:

Proposition 4. Given $M=\left(M_{x, y}^{a, b}\right)_{x, y=1, a, b=1}^{N, K}$, we have the following equivalence

$$
B_{C}(M) \leq\|M\|_{\ell_{1}^{N}\left(\ell_{\infty}^{K}\right) \otimes_{\epsilon} \ell_{1}^{N}\left(\ell_{\infty}^{K}\right)} \leq 4 B_{C}(M) .
$$


Proof. By duality, it follows that

$$
\|M\|_{\ell_{1}^{N}\left(\ell_{\infty}^{K}\right) \otimes_{\epsilon} \ell_{1}^{N}\left(\ell_{\infty}^{K}\right)}=\sup \left\{\sum_{a, x, b, y} M_{x, y}^{a, b} T_{x, y}^{a, b}: T_{x, y}^{a, b} \in B_{\ell_{\infty}^{N}\left(\ell_{1}^{K}\right) \otimes_{\pi} \ell_{\infty}^{N}\left(\ell_{1}^{K}\right)}\right\} .
$$

Since $B_{\ell_{\infty}^{N}\left(\ell_{1}^{K}\right) \otimes_{\pi} \ell_{\infty}^{N}\left(\ell_{1}^{K}\right)}$ is the convex hull of the set $\left\{x \otimes y: x \in B_{\ell_{\infty}^{N}\left(\ell_{1}^{K}\right)}, y \in\right.$ $\left.B_{\ell_{\infty}^{N}\left(\ell_{1}^{K}\right)}\right\}$, we have that

$$
\|M\|_{\ell_{1}^{N}\left(\ell_{\infty}^{K}\right) \otimes_{\epsilon} \ell_{1}^{N}\left(\ell_{\infty}^{K}\right)}=\sup \left\{\sum_{a, x, b, y} M_{x, y}^{a, b} \int_{\Omega} P_{\omega}(x, a) Q_{\omega}(y, b) d \mathbb{P}(\omega)\right\},
$$

where the sup is taken over all

a) $(\Omega, \mathbb{P})$ probability space,

b) $\sum_{a=1, \cdots, K}\left|P_{\omega}(x, a)\right| \leq 1$ for every $x=1, \cdots, N$ and every $\omega$,

c) $\sum_{b=1, \cdots, K}\left|Q_{\omega}(y, b)\right| \leq 1$ for every $y=1, \cdots, N$ and every $\omega$.

Using this, the first inequality follows. For the second one it is enough to consider the positive and negative part of each $P_{\omega}(x, a)$ and $Q_{\omega}(y, b)$.

Next we deal with the Quantum bound:

Theorem 6. Given $M=\left(M_{x, y}^{a, b}\right)_{x, y=1, a, b=1}^{N, K}$, we have the following equivalence

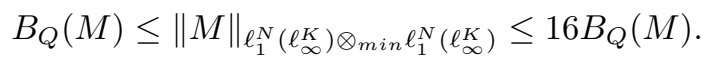

Before we prove the result, let us note that

$$
\|M\|_{\ell_{1}^{N}\left(\ell_{\infty}^{K}\right) \otimes_{\min } \ell_{1}^{N}\left(\ell_{\infty}^{K}\right)}=\sup \left\{\|(u \otimes v)(M)\|_{B(H) \otimes_{\min } B(H)}\right\}
$$

where the sup is taken over all the operators $u: \ell_{1}^{N}\left(\ell_{\infty}^{K}\right) \rightarrow B(H)$ which verify $\|u\|_{c b} \leq 1$ (and the same for $v$ ). We will use the following Lemma:

Lemma 2. Let $\left(T_{n}\right)_{n} \subset B(H)$ be a sequence of positive operators. Then $\left\|\sum_{n} T_{n}\right\|_{B(H)}=$ $\left\|\sum_{n} T_{n} \otimes e_{n}\right\|_{B(H) \otimes_{\text {min }} \ell_{1}}$, where we are considering the natural operator space structure on $\ell_{1}$.

Proof. It can be seen [46, Prop. 8. 9] that, for every sequence $\left(a_{n}\right)_{n}$ in $B(H)$, we have

$$
\left\|\sum_{n} a_{n} \otimes e_{n}\right\|_{B(H) \otimes_{m i n} \ell_{1}}=\inf \left\{\left\|\sum_{n} b_{n} b_{n}^{*}\right\|^{\frac{1}{2}}\left\|\sum_{n} c_{n}^{*} c_{n}\right\|^{\frac{1}{2}}\right\}
$$

where the inf is taken over all possible decompositions $a_{n}=b_{n} c_{n}$. Now, if we take $b_{n}=c_{n}=\left(T_{n}\right)^{\frac{1}{2}}$, we obtain

$$
\left\|\sum_{n} T_{n} \otimes e_{n}\right\|_{B(H) \otimes_{\min \ell_{1}}} \leq\left\|\sum_{n} T_{n}\right\|_{B(H)} .
$$


On the other hand, it is known [46, Prop. 8. 9] that the norm of $\| \sum_{n} T_{n} \otimes$ $e_{n} \|_{B(H) \otimes_{\min } \ell_{1}}$ is equal to

$$
\sup \left\{\left\|\sum_{n} T_{n} \otimes U_{n}\right\|_{B(H) \otimes_{\min } B(H)}: U_{n} \in B(H), U_{n} U_{n}^{*}=U_{n}^{*} U_{n}=\mathbb{1}\right\} .
$$

Then, taking $U_{n}=\mathbb{1}$ for every $n$, we get

$$
\left\|\sum_{n} T_{n}\right\|_{B(H)} \leq\left\|\sum_{n} T_{n} \otimes e_{n}\right\|_{B(H) \otimes_{m i n} \ell_{1}} .
$$

Alternatively, this follows from the fact that the functional $\Sigma: \ell_{1} \rightarrow \mathbb{C}, \Sigma\left(\left(t_{n}\right)\right)=$ $\sum_{n} t_{n}$ is a complete contraction.

The following remark will make the proof of Theorem 6 easier to read

Remark 2. Note that, using the isometric identification

$$
C B\left(\ell_{1}^{N}\left(\ell_{\infty}^{K}\right), B(H)\right)=\ell_{\infty}^{N}\left(\ell_{1}^{K}\right) \otimes_{\min } B(H)=\ell_{\infty}^{N}\left(\ell_{1}^{K} \otimes_{\min } B(H)\right),
$$

we can deduce from the previous lemma that $B_{Q}(M)$ is exactly the same as $\|M\|_{\ell_{1}^{K}\left(\ell_{\infty}^{N}\right) \otimes_{\min } \ell_{1}^{K}\left(\ell_{\infty}^{N}\right)}$, when we consider operators $u$ and $v$ which map the canonical basis of $\ell_{1}^{K}\left(\ell_{\infty}^{N}\right)$ to positive elements of $B(H)$ in Equation (13).

Indeed, this is immediate from the two following facts. First, given a complete contraction $u: \ell_{1}^{N}\left(\ell_{\infty}^{K}\right) \rightarrow B(H)$ such that $u\left(e_{x} \otimes e_{a}\right)=G_{x}^{a} \in B(H)^{+}$for every $x=1, \cdots, N ; a=1, \cdots, K$, we will have that, for every $x$,

$$
\left\|\sum_{a=1}^{K} G_{x}^{a}\right\|=\left\|\sum_{a=1}^{K} G_{x}^{a} \otimes e_{a}\right\|_{B(H) \otimes_{\min } \ell_{1}^{k}} \leq \sup _{x}\left\|\sum_{a=1}^{K} G_{x}^{a} \otimes e_{a}\right\|_{B(H) \otimes_{\min } \ell_{1}^{k}} \leq 1,
$$

where we have used Lemma 2 in the first equality and Equation (14) in the last inequality. $\sum_{a=1}^{K} G_{x}^{a}$ being a positive element for every $x$, the above estimation implies that we have a sequence of operators $\left(G_{x}^{a}\right)_{x=1, \cdots, N}^{a=1, \cdots, K} \subset B(H)^{+}$such that $\sum_{a=1}^{K} G_{x}^{a} \leq \mathbb{1}$ for every $x=1, \cdots, N$. On the other hand, given a sequence $\left(E_{x}^{a}\right)_{x=1, \cdots, N}^{a=1, \cdots, K} \subset B(H)^{+}$such that $\sum_{a=1}^{K} E_{x}^{a} \leq \mathbb{1}$ for every $x=1, \cdots, N$, we can consider the operator $u: \ell_{1}^{N}\left(\ell_{\infty}^{K}\right) \rightarrow B(H)$ defined by $u\left(e_{x} \otimes e_{a}\right)=E_{x}^{a} \in B(H)^{+}$. Using again Lemma 2 and Equation (14) we can see that

$$
\|u\|_{c b}=\sup _{x}\left\|\sum_{a=1}^{K} E_{x}^{a} \otimes e_{a}\right\|_{B(H) \otimes_{\min } \ell_{1}^{k}}=\sup _{x}\left\|\sum_{a=1}^{K} E_{x}^{a}\right\| \leq 1 .
$$

We can prove now Theorem 6 .

Proof (of Theorem [6). The first inequality is just the previous remark. For the proof of the second inequality we consider a complete contraction $u: \ell_{1}^{N}\left(\ell_{\infty}^{K}\right) \rightarrow$ $B(H)$. We consider now the $u_{x}: \ell_{\infty}^{K} \rightarrow B(H)$. We recall that according to Wittstock's factorization theorem [42, Theorem 8.5] every complete contraction $u$ defined on a $C^{*}$-algebra $\mathcal{A}$ with values in $B(H)$ can be decomposed as $u(x)=$ 
$V \pi(x) W$, with $V$ and $W$ contractions, and $\pi$ a ${ }^{*}$-representation. Thus, we have $u=u_{1}^{1}-u_{2}^{2}-i\left(u^{3}-u^{4}\right)$, where

$$
\begin{aligned}
& u_{1}^{1}(x)=1 / 4\left(V+W^{*}\right) \pi(x)\left(V^{*}+W\right), u_{2}^{2}(x)=1 / 4\left(V-W^{*}\right) \pi(x)\left(V^{*}-W\right), \\
& u_{3}^{3}(x)=1 / 4\left(V-i W^{*}\right) \pi(x)\left(V^{*}+i W\right), u^{4}(x)=1 / 4\left(V+i W^{*}\right) \pi(x)\left(V^{*}-i W\right)
\end{aligned}
$$

Note that, for every $i=1, \ldots, 4, u^{i}$ is a completely positive contraction.

We apply this observation to every component and we decompose $u_{x}=u_{x}^{1}-$ $u_{x}^{2}-i\left(u_{x}^{3}-u_{x}^{4}\right)$ as a linear combination of completely positive maps. Then, for every $x$ and $a$ we see that $\left(u_{x}^{i}\left(e_{a}\right)\right)_{a}=\left(E_{x, i}^{a}\right)_{a}$ is an incomplete POVM (see also Equation (14). This leads to the constant $16=4 \times 4$ in the assertion.

The following corollary follows now from the previous two theorems:

Corollary 4. Given $M=\left(M_{x, y}^{a, b}\right)_{x, y=1, a, b=1}^{N, K}$, we have that

$$
L V(M) \simeq \frac{\|M\|_{\ell_{1}^{N}\left(\ell_{\infty}^{K}\right) \otimes_{\min } \ell_{1}^{N}\left(\ell_{\infty}^{K}\right)}}{\|M\|_{\ell_{1}^{N}\left(\ell_{\infty}^{K}\right) \otimes_{\epsilon} \ell_{1}^{N}\left(\ell_{\infty}^{K}\right)}},
$$

where $\simeq$ denotes equality up to universal constants.

\section{Proof of the main result}

We introduce some notation that will be useful in the proof.

Remark 3. Given an operator space $X$, we construct an associated operator space $X^{n}$ as follows: Let $I$ be the collection of all complete contractions $v: X \longrightarrow M_{n}$. Then, we can define a new operator space structure on the Banach space $X$ considering the application

$$
j: X \longrightarrow \ell_{\infty}\left(I, M_{n}\right)
$$

defined by

$$
j(x)=\left((v(x))_{v \in I} .\right.
$$

It is easy to see that

$$
M_{n}\left(X^{n}\right)=M_{n}(X) .
$$

For our purpose it is interesting to note that

$$
\|a\|_{X^{n} \otimes_{\min } Y^{n}}=\sup _{\left\|v: X \longrightarrow M_{n}\right\|_{c b} \leq 1,\left\|w: Y \longrightarrow M_{n}\right\|_{c b} \leq 1}\|(v \otimes w)(x)\|_{M_{n} \otimes_{\min } M_{n}} .
$$

Then, the result that we will prove is

Theorem 7. Given $2<q<\infty$ and $n \in \mathbb{N}$, take $m$ such that $n^{\frac{q}{2}} \leq m \leq 2 n^{\frac{q}{2}}$ (for instance $m=\left[n^{\frac{q}{2}}\right]$ ) and denote $X=\ell_{1}^{m^{n}}\left(\ell_{\infty}^{n}\right)$. Then, we can find an element $x \in X \otimes X$ of rank $n$ such that $\|x\|_{X \otimes_{\epsilon} X} \leq D(q)$ and $\|x\|_{X^{n} \otimes_{\min } X^{n}} \geq n^{\frac{1}{2}-\frac{2}{q}}$.

Theorem 3 follows now from Theorem 7 and Corollary 4.

For reference purposes, we state next Chevet's inequality, which will be used often in the following. For a proof see [34]. 
Theorem 8 (Chevet's inequality). There exists a universal constant $b$ such that for every Banach spaces $E, F$ and every sequence $\left(g_{s, t}\right)_{s, t}$ of independent normalized gaussian random variables, we have

$$
\left\|\sum_{s, t} g_{s, t} x_{s} \otimes y_{t}\right\|_{E \otimes_{\varepsilon} F} \leq b w_{2}\left(\left(x_{s}\right)_{s} ; E\right)\left\|\sum_{t} g_{t} y_{t}\right\|_{F}+b w_{2}\left(\left(y_{t}\right)_{t} ; F\right)\left\|\sum_{s} g_{s} x_{s}\right\|_{E},
$$

where, given a sequence $\left(x_{s}\right)_{s}$ in a Banach space $X$, we use the notation $w_{2}\left(\left(x_{s}\right)_{s} ; X\right)$ for

$w_{2}\left(\left(x_{s}\right)_{s} ; X\right)=\left\|\sum_{s} x_{s} \otimes e_{s}\right\|_{X \otimes_{\epsilon} \ell_{2}}=\sup \left\{\left(\sum_{s}\left|x^{*}\left(x_{s}\right)\right|^{2}\right)^{\frac{1}{2}}: x^{*} \in X^{*},\left\|x^{*}\right\| \leq 1\right\}$.

We can take $b=1$ if the spaces are real, whereas $b=4$ if they are complex.

We will need the following three technical lemmas.

Lemma 3. Let $1<q<\infty, n \leq m$ and $\left(g_{i j}\right)_{i, j=1}^{n, m}$ a family of normalized gaussian random variables. Consider $X_{t}^{q}=t^{-\frac{1}{q}} K\left(t ; \ell_{\infty}^{m}, R^{m}+C^{m}, \ell_{1}^{m}\right)$ for $t=\frac{n}{m}$. Then,

$$
\mathbb{E}\left\|\sum_{i, j=1}^{n, m} g_{i j} e_{j} \otimes e_{i}: X_{t}^{q} \longrightarrow R_{n} \cap C_{n}\right\|_{c b} \leq K m^{1-\frac{1}{q}} n^{\frac{1}{q}} C(m, n)
$$

where $K$ is a universal constant and $C(m, n)=1+\frac{\sqrt{\log (m)}}{n}$.

Proof. It follows from equation 12 that

$$
\begin{aligned}
& \left\|a: X_{t}^{q} \longrightarrow R_{n}\right\|_{c b} \\
& =\|a\|_{R_{n}\left(X_{t}^{*}\right)} \leq t^{\frac{1}{q}} \max \left\{\|a\|_{R_{n}\left(\ell_{1}^{m}\right)}, t^{\frac{-1}{2}}\|a\|_{R_{n}\left(R_{m} \cap C_{m}\right)}, t^{-1}\|a\|_{R_{n}\left(\ell_{\infty}^{m}\right)}\right\} .
\end{aligned}
$$

where $c$ is a universal constant. We have to estimate the three terms appearing in this maximum. Recall our use of $\preceq$ for inequalities valid up to a universal constant. For the first term, we use the little Grothendieck theorem [25, Page 183], which says that there exists a constant $k$ such that for every operator $a: \ell_{\infty}^{m} \longrightarrow \ell_{2}^{n}$ we have $\left\|a: \ell_{\infty}^{m} \longrightarrow R_{n}\right\|_{c b} \leq k\|a\|_{o p}$ (and the same for $C_{m}$ ). Then, we invoke Chevet's inequality and obtain

$$
\mathbb{E}\left\|\sum_{i, j=1}^{n, m} g_{i j} e_{j} \otimes e_{i}\right\|_{R_{n}\left(\ell_{1}^{m}\right)} \preceq \mathbb{E}\left\|\sum_{i, j=1}^{n, m} g_{j, i} e_{i} \otimes e_{j}\right\|_{\ell_{1}^{m} \otimes_{\epsilon} \ell_{2}^{n}} \preceq(\sqrt{m} \sqrt{n}+m) \leq K_{1} m .
$$

For the second term, it is easy to see that

$$
\mathbb{E}\left\|\sum_{i, j=1}^{n, m} g_{i j} e_{j} \otimes e_{i}\right\|_{R_{n}\left(R_{m} \cap C_{m}\right)}=\mathbb{E}\left\|\sum_{i, j=1}^{n, m} g_{i j} e_{j} \otimes e_{i}\right\|_{\ell_{2}^{n} \otimes_{2} \ell_{2}^{m}} \preceq \sqrt{n m} .
$$


Finally, we will use Chevet's inequality again to estimate the last expression,

$$
\mathbb{E}\left\|\sum_{i, j=1}^{n, m} g_{i j} e_{j} \otimes e_{i}\right\|_{R_{n}\left(\ell_{\infty}^{m}\right)}=\mathbb{E}\left\|\sum_{i, j=1}^{n, m} g_{i j} e_{j} \otimes e_{i}\right\|_{\ell_{2}^{n} \otimes_{\epsilon} \ell_{\infty}^{m}} \preceq \sqrt{n}+\sqrt{\log m},
$$

where we have used that $\mathbb{E}\left\|\sum_{i=1}^{m} g_{i} e_{i}\right\|_{\ell_{\infty}^{m}} \preceq \sqrt{\log m}$ [54, Page 15]. Let us insert the precise value of $t=\frac{n}{m}$. Then we obtain

$$
\begin{aligned}
\mathbb{E} \| a & : X_{t}^{q} \longrightarrow R_{n} \|_{c b} \leq c t^{\frac{1}{q}} \mathbb{E}\left[\max \left\{\|a\|_{R_{n}\left(\ell_{1}^{m}\right)}, t^{\frac{-1}{2}}\|a\|_{R_{n}\left(R_{m} \cap C_{m}\right)}, t^{-1}\|a\|_{R_{n}\left(\ell_{\infty}^{m}\right)}\right\}\right] \\
& =c \mathbb{E}\left[\left(\frac{n}{m}\right)^{\frac{1}{q}} \max \left\{\|a\|_{R_{n}\left(\ell_{1}^{m}\right)},\left(\frac{n}{m}\right)^{\frac{-1}{2}}\|a\|_{R_{n}\left(R_{m} \cap C_{m}\right)},\left(\frac{n}{m}\right)^{-1}\|a\|_{R_{n}\left(\ell_{\infty}^{m}\right)}\right\}\right] \\
& \leq c \mathbb{E}\left[\left(\frac{n}{m}\right)^{\frac{1}{q}}\left(\|a\|_{R_{n}\left(\ell_{1}^{m}\right)}+\left(\frac{n}{m}\right)^{\frac{-1}{2}}\|a\|_{R_{n}\left(R_{m} \cap C_{m}\right)}+\left(\frac{n}{m}\right)^{-1}\|a\|_{R_{n}\left(\ell_{\infty}^{m}\right)}\right)\right] \\
& \leq K^{\prime}\left(\frac{n}{m}\right)^{\frac{1}{q}}\left(m+m+(\sqrt{n}+\sqrt{\log m})\left(\frac{m}{n}\right)\right) \leq K m^{1-\frac{1}{q}} n^{\frac{1}{q}}\left(1+\frac{\sqrt{\log (m)}}{n}\right),
\end{aligned}
$$

where $K$ is a universal constant. Replacing $R_{n}$ by $C_{n}$ we find the same estimates. By the definition of the intersection $R_{n} \cap C_{n}$ we obtain the result.

Lemma 4. There exits $\delta \in(0,1 / 2)$ with the following property: Given natural numbers $n \leq m$ and a family of normalized gaussian random variables $\left(g_{i j}\right)_{i, j=1}^{n, m}$, we consider $G=\frac{1}{\sqrt{m}} \sum_{i, j=1}^{n, m} g_{i j} e_{i} \otimes e_{j}$ as an operator from $\ell_{2}^{n}$ to $\ell_{2}^{m}$. Then, "with high probability", there exists an operator $v: H_{n} \longrightarrow \ell_{2}^{n}$ such that $\left.v \frac{1}{m} G^{*} G\right|_{H_{n}}=$ $\mathbb{1}_{H_{n}}$ and $\|v\| \leq 2$, where we denote $H_{n}=\ell_{2}^{[\delta n]}$.

Proof. Chevet's inequality tells us that

$$
\mathbb{E}\left[\|G\|_{o p}\right] \leq a \frac{1}{\sqrt{m}}(\sqrt{n}+\sqrt{m}) \leq C
$$

for some universal constant $C$. On the other hand, it is known [35, Page 80] that

$$
\mathbb{E}\left[\|G\|_{2}\right] \geq c \sqrt{n}
$$

for a universal constant $c>\frac{1}{\sqrt{2}}$, where $\|\cdot\|_{2}$ denotes the Hilbert-Schmidt norm. Thus, we can choose constants (independent of $n$ ) $0<c<C$ such that, with high probability, $G$ verifies $\|G\|_{o p} \leq C$, and $\|G\|_{2} \geq c \sqrt{n}$. We define $\delta=\frac{c^{2}}{2 C^{2}}$. We recall the notation $s_{j}(G)$ for the $j^{\text {th }}$ singular value of $G$ and observe that

$$
c^{2} n \leq\|G\|_{2}^{2}=\sum_{j=1}^{n} s_{j}(G)^{2} \leq s_{1}(G)^{2}([\delta n]-1)+s_{[\delta n]}(G)^{2} n \leq \frac{c^{2} n}{2}+s_{[\delta n]}(G)^{2} n .
$$

Therefore, we find $0<\frac{c^{2}}{2} \leq s_{[\delta n]}(G)^{2}$. We may take $c^{2}>\frac{1}{2}$, so we have $\frac{1}{2} \leq$ $s_{[\delta n]}(G)$. By the definition of the singular values of $G$, the above estimation says that we can invert the operator $\frac{1}{m} G^{*} G: \ell_{2}^{n} \longrightarrow \ell_{2}^{n}$ on a "large" subspace of dimension $k_{n}=[\delta n]$. Thus, if we denote $H_{n}=\ell_{2}^{[\delta n]}$, we know that there exists an operator $v_{n}: H_{n} \longrightarrow \ell_{2}^{n}$ such that $\left.v_{n} \frac{1}{m} G^{*} G\right|_{H_{n}}=\mathbb{1}_{H_{n}}$ and $\left\|v_{n}\right\| \leq 2$. 
Before we prove the next lemma, let us observe the following remark.

Remark 4. By the definition of the $K$-spaces and the standard interpolation equality $\left[\ell_{\infty}^{m}, \ell_{1}^{m}\right]_{1 / q}=\ell_{q}^{m}$, it is clear that for every $t>0, m \in \mathbb{N}$ and $1<$ $q<\infty$ the map id $=$ id o id $: \ell_{q}^{m} \hookrightarrow K\left(t ; \ell_{\infty}^{m}, \ell_{1}^{m}\right) \hookrightarrow K\left(t ; \ell_{\infty}^{m}, R^{m}+C^{m}, \ell_{1}^{m}\right)$ is a composition of two contractions (see for instance [5] for the first one), hence id is itself a contraction.

Lemma 5. Given $2<q<\infty$, there exists a constant $c(q)>0$ such that for every $n \leq m^{\frac{2}{q}}$ and every family of normalized gaussian random variables $\left(g_{i j}\right)_{i, j=1}^{n, m}$, we have

$$
\mathbb{E}\left\|m^{-\frac{1}{q}} \sum_{i, j=1}^{n, m} g_{i j} e_{i} \otimes e_{j}: \ell_{2}^{n} \longrightarrow X_{t}^{q}\right\| \leq c(q)
$$

(for every $t>0$ ).

Proof. Applying Chevet's inequality again for $X_{t}^{q}$, we get

$$
\mathbb{E}\left\|\sum_{i, j=1}^{n, m} g_{i j} e_{i} \otimes e_{j}\right\|_{\ell_{2}^{n} \otimes \epsilon X_{t}^{q}} \preceq \mathbb{E}\left\|\sum_{j=1}^{m} g_{j} e_{j}\right\|_{X_{t}^{q}}+\sqrt{n} w_{2}\left(\left(e_{j}\right)_{j=1}^{m} ; X_{t}^{q}\right) .
$$

Hence, it suffices to show that

$$
\begin{gathered}
\left\|i d: \ell_{2}^{m} \longrightarrow X_{t}^{q}\right\| \leq A(q) \text { and } \\
\mathbb{E}\left\|\sum_{j=1}^{m} g_{j} e_{j}\right\|_{X_{t}^{q}} \leq B(q) m^{\frac{1}{q}} .
\end{gathered}
$$

Both estimations follow easily using Remark 4. Indeed, the upper estimate follows, with $A(q)=1$, from

$$
\left\|i d: \ell_{2}^{m} \longrightarrow \ell_{q}^{m}\right\| \leq 1 .
$$

In the same way, the next estimate follows from

$$
\mathbb{E}\left\|\sum_{j=1}^{m} g_{j} e_{j}\right\|_{X_{t}^{q}} \leq \mathbb{E}\left\|\sum_{j=1}^{m} g_{j} e_{j}\right\|_{\ell_{q}^{m}} \leq B(q) m^{\frac{1}{q}} .
$$

Remark 5. It is well known that $B(q) \leq C \sqrt{q}$, where $C$ is a universal constant independent of $q$. Thus, we have that $c(q) \leq C^{\prime} \sqrt{q}$.

Using this, we can separate the epsilon and the min norm on a suitable subspace of $\ell_{1}^{m^{n}}\left(\ell_{\infty}^{n}\right)$.

Theorem 9. Given $2<q<\infty$ and $n \in \mathbb{N}$, if we take $n^{\frac{q}{2}} \leq m \leq 2 n^{\frac{q}{2}}$, there exists a matrix $a \in X_{t}^{q} \otimes X_{t}^{q}$ of rank $n$ such that $\|a\|_{\epsilon} \leq D(q)$ and $\|a\|_{\text {min }} \geq$ $n^{\frac{1}{2}-\frac{2}{q}}$, where we define $t=\frac{n}{m}$ and $X_{t}^{q}=t^{-\frac{1}{q}} K\left(t ; \ell_{\infty}^{m}, r^{m}+c^{m}, \ell_{1}^{m}\right)$. 
Proof. Given $n \in \mathbb{N}$ and $2<q<\infty$, taking $n^{\frac{q}{2}} \leq m \leq 2 n^{\frac{q}{2}}$, we define $t$ and $X_{t}^{q}$ as in the statement of the theorem. Since $t$ is considered fixed we may simplify the notation and write $X_{q}=X_{t}^{q}$. Thanks to the three previous Lemmas, we know that there exists a matrix $G=\left(g_{i j}(w)\right)_{i, j=1}^{n, m}$ such that

1) $\left\|G^{*}: X_{q} \longrightarrow R_{n} \cap C_{n}\right\|_{c b} \leq C(q) m^{\frac{1}{q^{\prime}}} n^{\frac{1}{q}}$.

2) There exist $\delta, v_{n}$ and $H_{n}$ as in Lemma 4

3) $\left\|G: \ell_{2}^{n} \longrightarrow X_{q}\right\| \leq c(q) m^{\frac{1}{q}}$.

Observe that, due to the choice of $m$, the function $C(n, m)=C(q)$ (in Lemma (3) only depends on $q$. Consider an arbitrary matrix $a$ in $H_{n} \otimes H_{n}$. Then, we have

$$
\left\|m^{-\frac{1}{q}} G \otimes m^{-\frac{1}{q}} G(a)\right\|_{X_{q} \otimes_{\epsilon} X_{q}} \leq c(q)^{2}\|a\|_{H_{n} \otimes_{\epsilon} H_{n}} .
$$

On the other hand, we have

$$
\begin{aligned}
\|a\|_{\ell_{2}^{n} \otimes_{2} \ell_{2}^{n}} & =\left\|v\left(\frac{1}{m} G^{*} G\right) \otimes v\left(\frac{1}{m} G^{*} G\right)(a)\right\|_{\ell_{2}^{n} \otimes_{2} \ell_{2}^{n}} \\
& =\left\|v\left(\frac{1}{m} G^{*} G\right) \otimes v\left(\frac{1}{m} G^{*} G\right)(a)\right\|_{R_{n} \cap C_{n} \otimes_{\min } R_{n} \cap C_{n}} \\
& \leq\left\|v m^{-\frac{1}{q^{\prime}}} G^{*}\right\|_{c b}^{2}\left\|\left(m^{-\frac{1}{q}} G \otimes m^{-\frac{1}{q}} G\right)(a)\right\|_{X_{q}^{n} \otimes_{\min } X_{q}^{n} .}
\end{aligned}
$$

In the special case where $a$ represents the identity on $H_{n}$ we obtain

$$
\begin{aligned}
\sqrt{n} & \leq \delta^{-\frac{1}{2}} \sqrt{k_{n}}=\delta^{-\frac{1}{2}}\|a\|_{\ell_{2}^{n} \otimes_{2} \ell_{2}^{n}} \\
& \leq 4 \delta^{-\frac{1}{2}} C(q)^{2} n^{\frac{2}{q}}\left\|\left(m^{-\frac{1}{q}} G \otimes m^{-\frac{1}{q}} G\right)(a)\right\|_{X_{q}^{n} \otimes_{\min } X_{q}^{n} .}
\end{aligned}
$$

This leads to the two competing estimates

$$
\begin{aligned}
\left\|m^{-\frac{1}{q}} G \otimes m^{-\frac{1}{q}} G(a)\right\|_{X_{q} \otimes_{\epsilon} X_{q}} & \leq c(q)^{2} \\
\left\|\left(m^{-\frac{1}{q}} G \otimes m^{-\frac{1}{q}} G\right)(a)\right\|_{X_{q}^{n} \otimes_{\min } X_{q}^{n}} & \geq \frac{D}{C(q)^{2}} n^{\frac{1}{2}-\frac{2}{q}} .
\end{aligned}
$$

Combing (16) and 17) yields the result.

Remark 6. According to Remark 3 , we have actually proved that

$$
\|a\|_{X_{q}^{n} \otimes_{\min } X_{q}^{n}} \geq \frac{D}{C(q)^{2}} n^{\frac{1}{2}-\frac{2}{q}} .
$$

Remark 7. The constant in the previous theorem can be taken $D(q) \leq C c(q)^{2} C(q)^{2}$, where $C$ is a universal constant which does not depend on $q$. Furthermore, we have seen in Remark 5 that $c(q)^{2} \preceq q$. It can be checked that $C(q) \leq 1+\frac{\sqrt{q \log (n)}}{n}$.

We can prove now Theorem 7 .

Proof. By Theorem 5 for every measure space $(\Omega, \mu)$ such that $\mu(\Omega)=k<$ $\infty$, we have that $L_{1}(\Omega)+L_{2}^{R}(\Omega)+L_{2}^{C}(\Omega)+L_{\infty}(\Omega)$ completely embeds into $L_{1}\left(\Omega^{k} ; \ell_{\infty}^{k}\right)$. Furthermore, the complete embedding

$$
j: L_{1}(\Omega)+L_{2}^{r}(\Omega)+L_{2}^{c}(\Omega)+L_{\infty}(\Omega) \hookrightarrow L_{1}\left(\Omega^{k} ; \ell_{\infty}^{k}\right)
$$


can be specifically written. Indeed, consider the measure space $(\Omega, \mu)$, where $\Omega=\{1, \cdots, m\}$ and $\mu(i)=t=\frac{n}{m}$ for every $i=1, \cdots, m$. Then, $\mu(\Omega)=m t=n$. But it is easy to see that for this measure space, the operator space $L_{1}(\Omega)+$ $L_{2}^{r}(\Omega)+L_{2}^{c}(\Omega)+L_{\infty}(\Omega)$ is exactly the operator space $K\left(t ; \ell_{\infty}^{m}, R^{m}+C^{m}, \ell_{1}^{m}\right)$. Thus, we have a completely isomorphic embedding of $K\left(t ; \ell_{\infty}^{m}, R^{m}+C^{m}, \ell_{1}^{m}\right)$ into $L_{1}\left(\Omega^{n}, \ell_{\infty}^{n}\right)$. Note that the difference between $L_{1}\left(\Omega^{n}, \ell_{\infty}^{n}\right)$ and $X=\ell_{1}^{m^{n}}\left(\ell_{\infty}^{n}\right)$ is just the normalization in the $L_{1}$-norm and hence the spaces are completely isometrically isomorphic. Thus, it will be enough to consider the completely isomorphic embedding $\tilde{j}=r \circ t^{-\frac{1}{q}} j$ from $t^{-\frac{1}{q}} K_{t}$ into $X$ and to take the element $x=(\tilde{j} \otimes \tilde{j})(a) \in X \otimes X$, where $a$ is the same element as in Theorem 9 . We invoke Remark 6 and the fact that the formal identity map $i d: X \rightarrow X^{n}$ is completely contractive. This yields the difference for the min and $\varepsilon$ norm claimed in the assertion.

Remark 8. It follows from Remark 7 that we can take $D(q) \leq q^{2}$ (actually, this estimate is not tight). Then, for a fixed dimension $n$, just taking $q=\log (n)$, we obtain

$$
\frac{\|x\|_{X^{n} \otimes_{\min } X^{n}}}{\|x\|_{X \otimes_{\epsilon} X}} \geq \frac{\sqrt{n}}{\log (n)^{2}}
$$

with $X=\ell_{1}^{\left[2^{\frac{\log ^{2} n}{2}}\right]^{n}}\left(\ell_{\infty}^{n}\right)$

Remark 9. We have the following interesting alternatives: either

a) for every subspace $F \subset L_{1}\left(\ell_{\infty}\right)$

$$
\ell_{2} \otimes_{\varepsilon} F=R+C \otimes_{\min } F
$$

or

b) there exists a subspace $F \subset L_{1}\left(\ell_{\infty}\right)$ such that

$$
\ell_{2} \otimes_{\varepsilon} F \neq R+C \otimes_{\min } F
$$

In case a), it follows easily from John's theorem [48] that for every rank $n$ tensor $a \in F_{1} \otimes F_{2}$ that

$$
\|a\|_{\min } \leq C \sqrt{n}\|a\|_{\varepsilon}
$$

This means our estimate 18 for a rank $n$ tensor is optimal up to the logarithmic factor. However, in case b) there are violations of Bell's inequality involving POVM's only for Alice or Bob, but not both. To wrap this up we could formulate it as follows. Either there are assymmetric Bell violations which are of simpler nature than everything discovered so far, or our estimates are best possible. It would certainly be interesting to know which of these alternatives holds true.

\section{Acknowledgments}

The authors are grateful to the organizers of the Operator Structures in Quantum Information Workshop, held in Toronto during July 6-10, 2009; where part of this work was developed. M. Junge is partially supported by the NSF grant DMS0901457. C. Palazuelos, D. Perez-Garcia and I. Villanueva are partially supported by Spanish grants I-MATH, MTM2008-01366 and CCG08-UCM/ESP4394. M.M. Wolf acknowledges support by QUANTOP and the Danish Natural Science Research Council(FNU). 


\section{References}

1. A. Acin, N. Brunner, N. Gisin, S. Massar, S. Pironio, V. Scarani, Device-independent security of quantum cryptography against collective attacks, Phys. Rev. Lett. 98, 230501 (2007).

2. A. Acin, N. Brunner, N. Gisin, S. Massar, S. Pironio, V. Scarani, Device-independent security of quantum cryptography against collective attacks, Phys. Rev. Lett., 98, 230501 (2007).

3. A. Acin, L. Masanes, N. Gisin, From Bell's Theorem to Secure Quantum Key Distribution, Phys. Rev. Lett. 97, 120405 (2006).

4. J.S. Bell, On the Einstein-Poldolsky-Rosen paradox, Physics, 1, 195 (1964).

5. C. Bennett, R. Sharpley, Interpolation of operators, Academic Press, 1988.

6. M. Ben-Or, A. Hassidim, H. Pilpel, Quantum Multi Prover Interactive Proofs with Communicating Provers, Proceedings of 49th Annual IEEE Symposium on Foundations of Computer Science (FOCS 2008), arXiv:0806.3982

7. G. Brassard, A. Broadbent, A. Tapp, Quantum Pseudo-Telepathy Foundations of Physics, Volume 35, Issue 11, Nov 2005, Pages 1877 - 1907.

8. J. Brit, H. Buhrman, B. Toner, A generalized Grothendieck inequality and entanglement in XOR games, arXiv:0901.2009

9. N. Brunner, N. Gisin, V. Scarani, C. Simon, Detection loophole in asymmetric Bell experiments, Phys. Rev. Lett., 98, 220403 (2007).

10. N. Brunner, S. Pironio, A. Acin, N. Gisin, A. A. Methot, V. Scarani, Testing the Hilbert space dimension, Phys. Rev. Lett. 100, 210503 (2008).

11. H. Buhrman, R. Cleve, S. Massar, R. de Wolf, Non-locality and Communication Complexity, to appear in Reviews of Modern Physics.

12. A. Cabello, J.-A. Larsson, Minimum detection efficiency for a loophole-free atom-photon Bell experiment, Phys. Rev. Lett. 98 (2007) 220402.

13. A. Cabello, D. Rodriguez, I. Villanueva, Necessary and sufficient detection efficiency for the Mermin inequalities, Rev. Lett. 101 (2008) 120402.

14. R. Cleve, P. Hyer, B. Toner, and J. Watrous, Consequences and Limits of Nonlocal Strategies, Proceedings of the 19th IEEE Annual Conference on Computational Complexity (CCC 2004), pp. 236- 249 (2004).

15. R. Cleve, D. Gavinsly, R. Jain,Entanglement-Resistant Two-Prover Interactive Proof Systems and Non-Adaptive Private Information Retrieval Systems, quant-ph/0707172 9, 2007.

16. A. Cohen, W. Dahmen and R. DeVore, Compressed sensing and best k-term approximation, JAMS, 22, No. 1,(2009), 211-231.

17. A. Defant and K. Floret, Tensor Norms and Operator Ideals, North-Holland, Amsterdam, 1993.

18. J. Degorre, M. Kaplan, S. Laplante, J. Roland, The communication complexity of nonsignaling distributions, arXiv:0804.4859

19. A. C. Doherty, Y-C. Liang, B. Toner, S. Wehner, The quantum moment problem and bounds on entangled multi-prover games, Proceedings of IEEE Conference on Computational Complexity 2008, pages 199-210.

20. E. G. Effros and Z.-J. Ruan, Operator spaces, London Math. Soc. Monographs New Series, Clarendon Press, Oxford, 2000.

21. A. Einstein, B. Podolsky, N. Rosen, Can Quantum-Mechanical Description of Physical Reality Be Considered Complete?, Phys. Rev., 47, 777 (1935).

22. A. Grothendieck, Résumé de la théorie métrique des produits tensoriels topologiques (French), Bol. Soc. Mat. So Paulo, 8, 1-79, (1953).

23. T. Holenstein, Parallel repetition: simplifications and the no-signaling case, Proceedings of the thirty-ninth annual ACM symposium on Theory of computing (STOC) 2007.

24. R. Jain, Z. Ji, S. Upadhyay, J. Watrous, QIP = PSPACE, arXiv:0907.4737

25. M. Junge, Factorization theory for Spaces of Operators, Habilitationsschrift Kiel, 1996; see also: Preprint server of the university of southern Denmark 1999, IMADA preprint: PP-1999-02.

26. M. Junge, J. Parcet, Rosenthal's theorem for subspaces of noncommutative Lp, Duke Math. J. 141, 75-122 (2008).

27. M. Junge, J. Parcet, Mixed-norm inequalities and operator space Lp embedding theory, To appear in Mem. Amer. Math. Soc.

28. M. Junge and J. Parcet, A transference method in quantum probability. Preprint.

29. M. Junge, J. Parcet and Q. Xu, Rosenthal type inequalities for free chaos, Ann. Probab. 35 (2007), 1374-1437. 
30. J. Kempe, O. Regev, B. Toner, The Unique Games Conjecture with Entangled Provers is False, Proceedings of 49th Annual IEEE Symposium on Foundations of Computer Science (FOCS 2008), quant-ph/0710.0655 (2007).

31. J. Kempe, H. Kobayashi, K. Matsumoto, B. Toner and T. Vidick, Entangled games are hard to approximate, arXiv:0704.2903 2 (2007).

32. S. Khot and N. K. Vishnoi. The unique games conjecture, integrality gap for cut problems and embeddability of negative type metrics into $\ell_{1}$. In Proc. 46th IEEE Symp. on Foundations of Computer Science, pages 53-62. (2005).

33. B. Kraus, N. Gisin, R. Renner, Lower and upper bounds on the secret key rate for QKD protocols using one-way classical communication, Phys. Rev. Lett. 95, 080501 (2005).

34. M. Ledoux, M. Talagrand, Probability in Banach Spaces, Springer-Verlag, 1991.

35. M.B. Marcus, G. Pisier, Random Fourier series with applications to Armonic Analysis, Annals of Math. Studies, 101, Princeton Univ. Press, (1981).

36. Ll. Masanes, R. Renner, A. Winter, J. Barrett, M. Christandl, Security of key distribution from causality constraints, quant-ph/0606049 (2006).

37. L. Masanes, Universally-composable privacy amplification from causality constraints, Phys. Rev. Lett. 102, 140501 (2009).

38. S. Massar. Nonlocality, closing the detection loophole, and communication complexity. Physical Review A, 65:032121, 2002.

39. S. Massar, S. Pironio, Violation of local realism vs detection efficiency, Phys. Rev. A 68, 062109 (2003).

40. M. Navascus, S. Pironio, A. Acn, Bounding the set of quantum correlations, Phys. Rev. Lett. 98, 010401 (2007)

41. M. Navascus, S. Pironio, A. Acn, A convergent hierarchy of semidefine programs characterizing the set of quantum correlations, New J. Phys. 10, 073013 (2008).

42. V. I. Paulsen, Completely Bounded Maps and Operator Algebras, Cambridge Studies in Advanced Mathematics 78, Cambridge University Press, Cambridge, 2003.

43. P. M. Pearle, Hidden-variable example based upon data rejection, Phys. Rev. D, 2:1418, 1970.

44. D. Pérez-García, M.M. Wolf, C. Palazuelos, I. Villanueva and M. Junge, Unbounded violation of tripartite Bell inequalities, Commun. Math. Phys. 279 (2), 455-486 (2008).

45. S. Pironio, Violations of Bell inequalities as lower bounds on the communication cost of nonlocal correlations. Physical Review A, 68(6):062102, 2003.

46. G. Pisier, An Introduction to Operator Spaces, London Math. Soc. Lecture Notes Series 294, Cambridge University Press, Cambridge 2003.

47. G. Pisier, Non-Commutative Vector Valued Lp-Spaces and Completely p-Summing Maps, Asterisque, 247 (1998)

48. G. Pisier, Factorization of linear operators and geometry of Banach spaces, CBMS 60 (1986).

49. , A. Rao, Parallel repetition in projection games and a concentration bound, STOC2008.

50. R. Raz, A Parallel Repetition Theorem, SIAM Journal on Computing 27, 763-803 (1998).

51. Z-J. Ruan, J. Funct. Anal. 76217 (1988).

52. Y. Shi and Y. Zhu. Tensor norms and the classical communication complexity of bipartite quantum measurements. SIAM Journal on Computing, 2008. To appear

53. P.W. Shor, J. Preskill, Simple Proof of Security of the BB84 Quantum Key Distribution Protocol, Phys. Rev. Lett. 85, 441-444, (2000).

54. N. Tomczak-Jaegermann, Banach-Mazur Distances and Finite Dimensional Operator Ideals, Pitman Monographs and Surveys in Pure and Applied Mathematics 38, Longman Scientific and Technical, 1989.

55. B.S. Tsirelson, Hadronic Journal Supplement 8:4, 329-345 (1993).

56. T. Vertesi, K.F. Pal, Bounding the dimension of bipartite quantum systems, arXiv:0812.1572

57. S. Wehner, M. Christandl, A. C. Doherty, A lower bound on the dimension of a quantum system given measured data, Phys. Rev. A 78, 062112 (2008).

58. R.F. Werner, M.M. Wolf, Bell inequalities and Entanglement, Quant. Inf. Comp., 1 no. 3, 1-25 (2001)

59. M.M. Wolf, D. Pérez-García, Assessing dimensions from evolution, Phys. Rev. Lett. 102, 190504 (2009). 


\section{A. Some proofs}

A.1. Proof of Proposition 2. The result is based on the fact that the norm of the identity

$$
i d: M_{n} \otimes_{\epsilon} M_{n} \rightarrow M_{n} \otimes_{\min } M_{n}
$$

is $\leq n$ (actually it is exactly $n$ ). Indeed, using that

$$
d_{c b}\left(R_{n}, \min \left(\ell_{2}^{n}\right)\right)=d_{c b}\left(C_{n}, \min \left(\ell_{2}^{n}\right)\right)=\sqrt{n},
$$

it is easy to see that $d_{c b}\left(M_{n}, \min \left(M_{n}\right)\right)=n$. The result follows now trivially from the fact $\min \left(M_{n}\right) \otimes_{\min } M_{n}=M_{n} \otimes_{\epsilon} M_{n}$.

Let us take then a Bell inequality $M=\left\{M_{x, y}^{a, b}\right\}_{x, y, a, b}$ and a quantum probability distribution $P$. By the previous estimation, we have

$$
\begin{aligned}
|\langle M, P\rangle| & \leq\left\|\sum_{a, b, x, y} M_{x, y}^{a, b} E_{a}^{x} \otimes F_{b}^{y}\right\|_{M_{n} \otimes_{\min } M_{n}} \leq \\
n & \left\|\sum_{a, b, x, y} M_{x, y}^{a, b} E_{a}^{x} \otimes F_{b}^{y}\right\|_{M_{n} \otimes_{\varepsilon} M_{n}} \cdot
\end{aligned}
$$

Now, this is exactly the same as

$$
\sup \left\{\left|\sum_{a, b, x, y} M_{x, y}^{a, b} \operatorname{tr}\left(E_{a}^{x} \rho_{1}\right) \operatorname{tr}\left(F_{b}^{y} \rho_{2}\right)\right|: \rho_{1}, \rho_{2} \in B_{S_{1}^{n}}\right\} .
$$

But it is well known that every $\rho \in B_{S_{1}^{n}}$ can be written as $\rho=\rho_{1}^{1}+i \rho_{1}^{2}$ with $\rho_{1}^{i}$ self adjoint elements in $B_{S_{1}^{n}}$ for $i=1,2$. Then,

$$
19 \leq 4 \sup \left\{\left|\sum_{a, b, x, y} M_{x, y}^{a, b} \operatorname{tr}\left(E_{a}^{x} \rho_{1}\right) \operatorname{tr}\left(F_{b}^{y} \rho_{2}\right)\right|: \rho_{1}, \rho_{2} \in B_{S_{1}^{n}} \text { and self adjoint }\right\} .
$$

But $\rho_{1}$ can be written as $\rho_{1}=\sum_{j=1}^{n} \delta_{j}\left|f_{j}\right\rangle\left\langle f_{j}\right|$ with $\left(\left|f_{j}\right\rangle\right)_{j}$ an orthonormal basis of $\ell_{2}^{n}$, and $\sum_{j=1}^{n}\left|\delta_{j}\right| \leq 1$ (and the same for $\rho_{2}$ ). Then, for every pair of selfadjoint $\rho_{1}, \rho_{2}$ we have

$$
\begin{aligned}
& \left|\sum_{a, b, x, y} M_{x, y}^{a, b} \operatorname{tr}\left(E_{a}^{x} \rho_{1}\right) \operatorname{tr}\left(F_{b}^{y} \rho_{2}\right)\right| \\
& \leq \sup \left\{\left|\sum_{a, b, x, y} M_{x, y}^{a, b}\left\langle u\left|E_{a}^{x}\right| u\right\rangle\left\langle v\left|F_{b}^{y}\right| v\right\rangle\right|:|u\rangle,|v\rangle \in S_{\ell_{2}^{n}}\right\},
\end{aligned}
$$

which is bounded above by $\sup _{P^{\prime} \in \mathcal{L}}\left|\left\langle M, P^{\prime}\right\rangle\right|$. Therefore, we have

$$
|\langle M, P\rangle| \preceq n \sup _{P^{\prime} \in \mathcal{L}}\left|\left\langle M, P^{\prime}\right\rangle\right| \text {. }
$$


A.2. Explanation of [[28], Theorem 3.6]. Suppose we have a probability space $(\Omega, \mu)$ and $k \in \mathbb{N}$. We may consider the particular case of (Theorem 3.6, 28]) in which $\mathcal{A}=M_{k} \otimes_{\min } L_{\infty}\left(\Omega^{n}\right), \mathcal{M}=M_{k} \otimes_{\min } L_{\infty}(\Omega), \mathcal{N}=M_{k}$, the conditional expectation $\mathcal{E}_{\mathcal{N}}: \mathcal{M} \rightarrow \mathcal{N}$ is defined by $\mathcal{E}_{\mathcal{N}}=\mathbb{1} \otimes \int \cdot d \mu$ and $\mathcal{K}=\mathbb{C}$. The algebras $(\mathcal{M})_{k \geq 1}$ 's form a system of independent symmetric system of copies of $\mathcal{M}$ over $\mathcal{N}$ (see $([28$, Example 1), which is a stronger condition than the one appearing in (Theorem 3.6, 28, ). We start with the easy case

$$
L_{1}\left(\mathcal{A}, \ell_{\infty}^{n}\right)=L_{1}\left(M_{k} \otimes L_{\infty}\left(\Omega^{n}\right), \ell_{\infty}^{n}\right)=S_{1}^{k}\left(L_{1}\left(\Omega^{n}\right), \ell_{\infty}^{n}\right) .
$$

Let us turn to the more complicated $\mathbb{K}$ space

$$
\mathcal{K}_{1, \infty}^{n}\left(\mathcal{M}, \mathcal{E}_{\mathcal{N}}\right)=n L_{1}(\mathcal{M})+L_{1}^{s}\left(\mathcal{M}, \mathcal{E}_{\mathcal{N}}\right)+\sqrt{n} L_{1}^{r}\left(\mathcal{M}, \mathcal{E}_{\mathcal{N}}\right)+\sqrt{n} L_{1}^{c}\left(\mathcal{M}, \mathcal{E}_{\mathcal{N}}\right) .
$$

Here we refer to definition before ([28], Lemma 3.5)

$$
\|x\|_{L_{1}^{s}\left(\mathcal{M}, \mathcal{E}_{\mathcal{N}}\right)}=\inf _{x=a y b}\|a\|_{L_{2}\left(M_{k}\right)}\|y\|_{M_{k} \otimes_{\min } L_{\infty}(\Omega)}\|b\|_{L_{2}\left(M_{k}\right)}=\|x\|_{S_{1}^{k}\left(L_{\infty}(\Omega)\right)} .
$$

Hence $L_{1}^{s}(\mathcal{M})=S_{1}^{k}\left(L_{1}(\Omega)\right)$ as predicted. For the column term we have

$$
\begin{aligned}
\|x\|_{L_{1}^{c}\left(\mathcal{M}, \mathcal{E}_{\mathcal{N}}\right)} & =\inf _{x=a y b}\|a\|_{L_{2}(\mathcal{M})}\|y\|_{M_{k} \otimes L_{\infty}(\Omega)}\|b\|_{L_{2}\left(M_{k}\right)} \\
& =\inf _{x=a b}\|a\|_{L_{2}(\mathcal{M})}\|b\|_{L_{2}\left(M_{k}\right)} .
\end{aligned}
$$

Given such a factorization $x=a b$ we see that

$$
\begin{aligned}
\left\|\left(\int_{\Omega}|x|^{2} d \mu\right)^{1 / 2}\right\| & =\left\|b^{*}\left(\int_{\Omega} a^{*} a d \mu\right) b\right\|_{1 / 2}^{2} \\
& \leq\|b\|_{L_{2}\left(M_{k}\right)}\left\|\int_{\Omega} \operatorname{tr}\left(a^{*} a\right) d \mu\right\|_{1}^{1 / 2}=\|b\|_{2}\|a\|_{2}
\end{aligned}
$$

This shows $\|x\|_{S_{1}^{k}\left(L_{2}^{r}(\Omega)\right)} \leq \inf \|a\|\|b\|$. Conversely, for $x \in S_{1}^{k}\left(L_{2}^{r}(\Omega)\right)=R_{k} \otimes_{h}$ $L_{2}^{r}(\Omega) \otimes_{h} C_{k}$ we deduce from the definition of the Haagerup tensor product that we can find a factorization $x=b a$ such that $b \in R_{k} \otimes_{h} L_{2}^{r}(\Omega) \otimes_{h} R_{k}$ and $a \in L_{2}\left(M_{k}\right)$. Note however, that

$$
\|b\|_{R_{k} \otimes_{h} L_{2}^{r}(\Omega) \otimes_{h} R_{k}}=\|b\|_{L_{2}\left(\Omega, S_{2}^{k}\right)}=\|b\|_{L_{2}(\mathcal{M})} .
$$

Thus we have in fact

$$
\|x\|_{S_{1}^{k}\left(L_{2}^{r}(\Omega)\right)}=\|x\|_{L_{1}^{c}\left(\mathcal{M}, \mathcal{E}_{\mathcal{N}}\right)}
$$

Interchanging rows and columns yields the missing estimate. Theorem 5 follows now easily. Suppose we have a measure space $(\Omega, \mu)$ such that $\mu(\Omega)=n$. Then, we consider $(\Omega, \hat{\mu})=\left(\Omega, \frac{\mu}{n}\right)$ and, thus,

$$
i: n L_{1}(\Omega, \hat{\mu})+\sqrt{n} L_{2}^{r}(\Omega, \hat{\mu})+\sqrt{n} L_{2}^{c}(\Omega, \hat{\mu})+L_{\infty}(\Omega, \hat{\mu}) \hookrightarrow L_{1}\left(\Omega^{n}, \otimes^{n} \hat{\mu} ; \ell_{\infty}^{n}\right),
$$

is a completely embedding. But it is obvious that

$$
\begin{gathered}
n L_{1}(\Omega, \hat{\mu})=L_{1}(\Omega, \mu), \sqrt{n} L_{2}^{r}(\Omega, \hat{\mu})=L_{2}^{r}(\Omega, \mu), \sqrt{n} L_{2}^{c}(\Omega, \hat{\mu})=L_{2}^{c}(\Omega, \mu), \\
L_{\infty}(\Omega, \hat{\mu})=L_{\infty}(\Omega, \mu) \text { and } n^{n} L_{1}\left(\Omega^{n}, \otimes^{n} \hat{\mu} ; \ell_{\infty}^{n}\right)=L_{1}\left(\Omega^{n}, \otimes^{n} \mu ; \ell_{\infty}^{n}\right) .
\end{gathered}
$$

Therefore,

$$
j=\frac{i}{n^{n}}: L_{1}(\Omega, \mu)+L_{2}^{r}(\Omega, \mu)+L_{2}^{c}(\Omega, \mu)+L_{\infty}(\Omega, \mu) \hookrightarrow L_{1}\left(\Omega^{n}, \otimes^{n} \mu ; \ell_{\infty}^{n}\right)
$$

is a complete embedding (with absolute constants). 